\title{
26 Research Square \\ Functional study of early intervention for degenerative retinopathy through hydrogel sustained release system of rhEPO
}

\section{Zhimeng Zhang}

Beijing Tongren Hospital

Hehua Dai

Beijing Tongren Hospital

Ruyi Li

Beijing Tongren Hospital

Yuyu Li

Beijing Tongren Hospital

Genlin Li ( $\nabla$ ligenlin2018@163.com )

Beijing Tonren Hospital

Research article

Keywords: hydrogel sustained release system, rhEPO, retinitis pigmentosa, subconjunctival injection, retrobulbar injection

Posted Date: January 24th, 2020

DOI: https://doi.org/10.21203/rs.2.16534/v3

License: (c) (1) This work is licensed under a Creative Commons Attribution 4.0 International License. Read Full License 


\section{Abstract}

Background Retinitis pigmentosa (RP) is the most common cause of blindness in retinal disease. Longlasting ocular administration is an effective therapy to delay the progression of RP. And hydrogel sustained release system may be an available and stable drug carrier in the treatment of RP. Method Hydrogel sustained release system was constructed as a drug carrier of recombinant human erythropoietin (rhEPO). We administered retinal degenerative (rd) mice (Pdeb rd1 / Pdeb rd1 ) via subconjunctival or retrobulbar injection at postnatal 2 weeks (PN-2w), examined the mice and tested the factors of retina at two weeks after injection. Electroretinogram (ERG) was used to examine retinal function at PN-4w, western blot and q-PCR were used to test the expression of Bax, Bcl-2, iNOS and VEGFa of retina. Result Photoreceptor apoptosis were alleviated in all rhEPO administrated groups. The retinal blood supply was improved in injection groups. Compared with placebo and blank control groups, rhEPO treatment could enhance the retinal function and delay the progression of disease. Although there was no significant difference between rhEPO hydrogel and rhEPO treated group, photoreceptor apoptosis in rhEPO hydrogel group was less than that in rhEPO group, and the retinal function was better in rhEPO hydrogel group. Moreover, different routes of administration might have little effect on treatment in this research. Conclusion Early intervention can effectively control the progression of the disease. Antiapoptosis,neuroprotection and erythropoietin of rhEPO could be useful in the treatment of RP. Hydrogel as a long-lasting drug sustained release system was stable and available, and might become a potential drug carrier in the future.

\section{Background}

Retinitis pigmentosa (RP) is a group of heretogeneous retinal degenerative disease that is characterized by progressive retinal pigment epithelium (RPE) and photoreceptor degeneration. Nyctalopia, progressive peripheral visual field deterioration, and blindness are its typical clinical manifestations. The prevalence of RP is about 1 in $3000-4000^{[1]}$. Nowadays, various therapeutic strategies have been made to against RP: gene therapy can correct genetic defects fundamentally to produce normal genotype-phenotype in targeted cells and express stably; drugs can delay the progression of RP through protecting photoreceptor cells against apoptosis; stem cell transplantation and retinal transplantation were used to replace the lesion area in the late stage of RP. Currently, gene therapy of RP has been made a breakthrough in animal models. It's worth to mention that the treatment of RPE65-mediated hereditary retinal dystrophy by AAV2hRPE65v2 has been successful in phase 3 clinical trials ${ }^{[2]}$. However, the pathogenesis of RP is complex, since there are 190 genes and nearly 3100 gene mutations have been proved to be associated with RP [3]. Therefore, further experimental studies are needed to treat RP fundamentally by gene therapy.

Furthermore, the low survival rate and migration rate of stem cells and the rejection of transplanted retina are all the problems in stem cell or retinal transplantation. Taking the above situations into consideration, drug therapy is still the main method of treating RP.

Erythropoietin (EPO) is consisted of 165-amino acid glycoprotein with a molecular weight of $30.4 \mathrm{kDa}$, which has multiple effects of hormones, cytokines and growth factors. It was only thought to be a 
glycoprotein produced in fetal liver and adult kidney initially, which mainly regulates erythropoiesis ${ }^{[4]}$. Now, it is confirmed that EPO is expressed in liver, kidney, nerve tissue, female genital tract, placenta, testis and retinas of adult mammals, and these tissues also express EPO receptors (EPOR) ${ }^{[5]}$. Previous studies suggested that in addition to hematopoiesis, anti-oxidation, anti-inflammation and anti-apoptosis are also effects of EPO ${ }^{[6-8]}$. EPO is expressed in ganglion cells, inner plexiform layer (IPL), outer plexiform layer (OPL) and horizontal cells of embryonic retina, and EPOR is expressed in ganglion cells layer (GCL) and most membranous tissues such as inner limiting membrane (ILM). It indicates that EPO plays an important role in the development of embryonic retina and in the repair and maintenance of mature retina ${ }^{[9]}$. From many preclinical studies, EPO may become a useful agent for various ocular diseases ${ }^{[10]}$, for example, decreasing the apoptosis of RGCs in glaucoma, reducing retinal blood vessel degeneration to against the progression in diabetic retinopathy (DR), and preventing RPE apoptosis due to oxidative damage in age-related macular degeneration (AMD). A current study showed that subretinal injection of EPO could effectively alleviate photoreceptor degeneration of N-methyl-N-nitrosourea (MNU) administered mice ${ }^{[11]}$. This result indicated that EPO would become a potential therapeutic strategy in treating RP.

Hydrogel is a copolymer with a three-dimensional network structure formed by cross-linking through covalent bonds, hydrogen bonds or van der Waals forces, etc., which can swell in water and retain a large amount of water. In the last decade, hydrogel as a sustained release system for ocular long-lasting administration has become a hotspot. The results from a previous study about a latanoprost-loaded chitosan-gelatin-based hydrogel for glaucoma treatment showed that this sustained release system can decrease intraocular pressure (IOP) within 8 days, and remain normal for at least 30 days after only one time subconjunctival injection ${ }^{[12]}$. Its non-toxicity to cells was proved in in vitro cytotoxicity tests ${ }^{[13]}$. Gao etc. successfully synthesized thermosensitive PLGA-PEG-PLGA, and indicated that this kind of hydrogel could enhance bioavailability of drugs significantly ${ }^{[14]}$. The hydrogel we used in present research, which was constructed of chitosan (CS) and polyethylene glycol (PEG), has already been used in the treatment of glaucoma, its sustained IOP lowering effect has been proved ${ }^{[15]}$. Thus, we highly believed that hydrogel as a drug delivery carrier could maintain the effect of drugs and control the release rate to achieve sustained-release.

Animal models are essential for experimental research. The rd mice (Pdeb $\left.{ }^{\text {rd1 } 1} \mathrm{Pdeb}^{\text {rd1 }}\right)$ with homozygous mutations were used as RP animal models of this study. Photoreceptor apoptosis of rd mice was caused by mutations in gene encoding the $\beta$-subunit of cGMP phosphodiesterase, which inducing rapid and massive death of rod cells in the first few weeks of life ${ }^{[16-17]}$. And the same gene mutation has also been found in human adRP ${ }^{[18]}$. In our previous studies about morphological changes of photoreceptor segments at early stage in hereditary retinal degenerative animal models ${ }^{[19]}$, we found that photoreceptor segments of rd mice were shorter than normal mice at postnatal 1 week ( $P N-1 w)$, these segments were undeveloped, irregular arrangement and began to degenerate at $\mathrm{PN}-2 \mathrm{w}$, apoptosis of photoreceptor segments peaked when rd mice were 3 weeks old, finally, the segment layer of rd mice were almost 
disappeared at $\mathrm{PN}-4 \mathrm{w}$. Therefore, our animal models showed characteristics like photoreceptor cells early-onset degeneration and rapid progression.

In this study, we used the rd mice (Pdeb ${ }^{\text {rd1 } / ~ P d e b ~}{ }^{\text {rd1 }}$ ) as RP animal models, constructed hydrogel sustained release system as a drug carrier of recombinant human erythropoietin (rhEPO) for RP treatment at the early stage. After a single subconjunctival injection, we investigated the changes of retinal function in each group of mice, to explore the role of EPO treatment in alleviating photoreceptor degeneration and make an assessment about the availability and stability of hydrogel sustained release system in drug delivery, so as to seek a long-lasting drug delivery system for the treatment of RP in the future.

\section{Methods}

\section{Synthesis of rhEPO/PBS hydrogel}

Glycol chitosan (CS, 430kDa) was purchased from FUJIFILM Wako Pure Chemical Corporation (Tokyo, Japan). Telechelic difunctional poly (ethylene glycol, DF-PEG-DF, 4000kDa) was purchased from HuaTeng Pharmaceutical Co. Ltd. (Hunan, China). Recombinant human erythropoietin (rhEPO, $10000 \mathrm{U} / \mathrm{ml}$ ) was purchased from 3S Bio (ShenYang, China). Add Chitosan powder into rhEPO injection or PBS buffer to obtain chitosan solution with a concentration of $10 \mathrm{mg} / \mathrm{ml}$. Add DF-PEG-DF powder into rhEPO injection or PBS buffer to obtain PEG solution with a concentration of $50 \mathrm{mg} / \mathrm{ml}$. The two solutions were mixed together with a ration of 1:1 at room temperature, after several minutes, the mixture solidified gradually and then form a hydrogel (Figure 1).

\section{Experimental animals and administration}

The rd (Pdeb ${ }^{\text {rd1 } / ~ P d e b ~}{ }^{\text {rd1 }}$ ) mice and the C57BL/ 6 mice (male and female unlimited, 4 weeks old) were used. The C57BL/6 mice were purchased from HFK Bioscience Co. Ltd (Beijing, China). The rd (Pdeb ${ }^{\text {rd1/ }}$ $\mathrm{Pdeb}^{\mathrm{rd} 1}$ ) mice were purchased from The Jackson Laboratory (USA) and housed in the experimental facility (room temperature at about $25^{\circ} \mathrm{C}$, on a 12-hour light/dark cycle) at the department of laboratory animal, Capital Medical University. The breeding grade was SPF (Specific Pathogen Animal). The care and maintenance of the mice were referred to the ARVO guidelines. The experiment was approved by the Animal Experiment Ethics Committee of Capital Medical University (NO. AEEI-2019-033). The mice were randomly divided into 8 groups, each group had 5 mice: (1) rhEPO hydrogel treated group (rhEPO hydrogel): mice in this group received subconjunctival injection (Figure 2) of 10ul rhEPO hydrogel at PN$2 \mathrm{w}$; (2) rhEPO treated group (rhEPO): mice in this group received subconjunctival injection of 10ul rhEPO at PN-2w; (3) placebo treated group 1 (PBS hydrofel): mice in this group received subconjunctival injection of 10ul PBS hydrogel at PN-2w; (4) placebo treated group 2 (PBS): mice in this group received subconjunctival injection of $10 \mathrm{ul} \mathrm{PBS}$ at PN-2w; (5) blank control group (RD): rd mice in this group were left without any treatment; (6) normal control group (C57): C57BL/6 mice in this group were left without any treatment. Two weeks after injections, the mice were examined and killed, and the retinas were taken 
for assay. The mice were euthanized by an overdose of anesthesia ( $0.3 \%$ sodium pentobarbital, $0.15 \mathrm{ml} / 10 \mathrm{~g}$, intraperitoneal injection).

\section{Western blot}

The retinas for 8 groups were lysed in RIPA buffer (Cell Signaling Technology, USA) mixed with protease inhibitor cocktail (MedChemExpress, USA) at a ratio of 100:1 on ice. The samples were treated with ultrasonic wave for 10 seconds. BCA protein assay kit (Beyotime, China) was used to measure protein concentrations. According to the molecular weight of target proteins $₫$ we made $6 \%$ and $12 \%$ SDSpolyacrylamide gels. 40ug proteins were added to each well. After electrophoresis, the proteins on the SDS-polyacrylamide gels were transferred onto polyvinylidene difluoride (PVDF) membranes. The membranes were blocked with $5 \%$ non-fat milk at room temperature for 1 hour and then incubated with primary antibody: Bax (1:1000, CST, USA, NO. 14796), Bcl-2 (1:1000, CST, USA, NO. 3498), or GAPDH (1:1000, CST, USA, NO. 5174) overnight at $4{ }^{\circ} \mathrm{C}$ or iNOS (1:600, Proteintech, USA, NO. 18985-1-AP), VEGFa (1:1500, Proteintech, USA, NO. 26157-1-AP) for $1.5 \mathrm{~h}$ at room temperature. After being washed with Tris-Buffered Saline and Tween 20 (TBST) for 3 times, the membranes were incubated with antirabbit secondary antibody (1:2000, CST, USA, NO. 7074) at room temperature for 1 hours, followed by another 3-time washes with TBST. Finally, the protein bands were shown using an ECL imager (Millipore, USA) and their values were analysed by BIO-RAD Quantity One Imaging software (Bio-Rad, USA). Western blot was normalized by GAPDH.

\section{Quantitative reverse transcription-polymerase chain reaction (qRT- PCR)}

Total RNA was extracted from mouse retinas, using chloroform and isopropyl alcohol. Reverse transcription (RT) was performed by using RT Master Mix for qPCR (MedChemExpress, USA) and realtime PCR was carried out by using SYBR Green qPCR Master Mix (MedChemExpress, USA). Reactions were performed in a CFX96 real-time system (Bio-Rad Laboratories, Switzerland). The amplification program consisted of polymerase activation at $95.0^{\circ} \mathrm{C}$ for $10 \mathrm{~min}$ and 40 cycles of polymerase activation at $95.0^{\circ} \mathrm{C}$ for $15 \mathrm{~s}$, primer annealing and extension at $60.0^{\circ} \mathrm{C}$ for $60 \mathrm{~s}$. The primers were designed on https://blast.ncbi.nlm.nih.gov/Blast.cgi and were ordered from Sangon Biotech (Shanghai, China). The primers for Bax were 5'-GCACGTCCACGATCAGTCA-3' (forward) and 5'-

CGATCCTGGATGAAACCCTGT-3' (reverse); the primers for Bcl-2 were 5'-GATAACGGAGGCTGGGATGC-3' (forward) and 5'-TCACTTGTGGCCCAGGTATG-3' (reverse); the primers for iNOS were 5'TCCTGGACATTACGACCCCT-3' (forward) and 5'-CTCTGAGGGCTGACACAAGG-3' (reverse); the primers for VEGFa were 5'-AACGAACGTACTTGCAGATGTGA-3' (forward) and 5'-CTGTCAACGGTGACGATGATG$3^{\prime}$ (reverse). GAPDH was used as the internal standard of mRAN expression.

\section{Electroretinogram (ERG) recording}


Two weeks after administration, the 12-hour dark-adapted mice were anesthetized by $0.3 \%$ sodium pentobarbital $(0.1 \mathrm{ml} / 10 \mathrm{~g}$, intraperitoneal injection). Their pupils were dilated with tropicamide phenylephrine eye drops (Santen Pharmaceutical Co., Ltd, Japan). The anesthetized mice were removed to the recording platform under the dark red light. Their cornea was anesthetized with oxybuprocaine hydrochloride eye drops (Santen Pharmaceutical Co., Ltd, Japan). ERG was performed by the RETIport system (Roland Consult, Brandenburg, Germany) with Ganzfield Q450 stimulator, a new light emitting diode (LED) flash stimulator, which standard white flash intensity is $3.0 \mathrm{CD} \cdot \mathrm{s} / \mathrm{m} 2$ when $0 \mathrm{~dB}$, and standard flash intensity attenuation is $2.5 \mathrm{log}$ units when $-25 \mathrm{db}$. The loop metal electrodes were fixed on the surface of the cornea to serve as recording electrodes. Reference and ground electrodes were inserted into cheeks and tail. After installation\we recorded scotopic 0.01 ERG, scotopic 3.0 and 10.0 ERG, scotopic 3.0 socillatory potentials, photopic 3.0 ERG and photopic 3.0 fliker and analyzed b-wave of scotopic 0.01 ERG and scotopic 3.0 and 10.0 ERG.

\section{Statistical analysis}

Data were expressed as the mean \pm standard deviation (SD). One-way ANOVA, Kruskal-Wallis one-way ANOVA and Independent samples $t$ test were used to determine the significant difference between different animal groups. $P<0.05$ was considered as statistically significant.

\section{Results}

rhEPO could alleviate the apoptosis of retinal photoreceptors and protect retinal neruons of rd mice

The mRNA and protein expression level of Bax in RD group were significantly higher than normal ( $p=$ $0.012, p=0.043$ ) (Figure 1A, Figure 2 and Figure $3 A$ ). There was no statistically significant difference between placebo groups and RD group. The mRNA and protein expression in rhEPO administration groups were obviously decreased, compared with RD group (rhEPO hydrogel: $p=0.018, p=0.032$; rhEPO: $p=0.047, p=0.043$ ). Although the mRNA and protein expression levels in rhEPO hydrogel group were lower than that in rhEPO group, there was no statistically significant difference between the two groups.

The mRNA and protein expression levels of $\mathrm{Bcl}-2$ in RD group were significantly lower than normal levels $(p=0.005, p=0.0 .047)$ (Figure 1B, Figure 2 and Figure 3B). The expression levels in two placebo groups were almost at the same level with RD group. Compared with RD group, the mRNA and protein expression in rhEPO hydrogel group were obviously increased $(p=0.039, p=0.002)$. And the mRNA and protein expression in rhEPO group were also significantly higher than that in $\operatorname{RD}$ group $(p=0.047, p=0.012)$. Although the expression levels in rhEPO hydrogel group were higher than those in rhEPO group, there was no statistically significant difference between the two groups.

The mRNA and protein expression levels of iNOS in RD groups were significantly higher than those in C57 group ( $p=0.046, p=0.027$ ) (Figure 1C, Figure 2 and Figure $3 C$ ). There was no statistically significant difference between placebo groups and RD group. The mRNA and protein expression in rhEPO 
administration groups were obviously decreased, compared with RD group (rhEPO hydrogel: $p=0.027, p$ $=0.005$; EPO: $p=0.045, p=0.022$ ). The expression levels in rhEPO hydrogel group were lower than those in rhEPO group, but there was no statistically significant difference between the two groups.

\section{rhEPOcould improve blood supply to the retina of rd mice}

The mRNA and protein expression levels of VEGFa in RD group were significantly lower than normal level $(p=0.039, p=0.012)$ (Figure 1D, Figure 2 and Figure 3D). There was no statistically significant difference between placebo groups and RD group. Compared with RD group, the mRNA and protein expression of VEGFa was significantly increased in rhEPO administration groups (rhEPO hydrogel: $p=0.025, p=0.044$; EPO: $p=0.022, p=0.018$ ).

\section{rhEPO could protect retinal function of rd mice}

Figure 4 was the results of $\mathrm{b}$ waves of scotopic $0.01 \mathrm{ERG}$. Figure $4 \mathrm{~A}$ represented the normal waveform in C57 group. The value of $b$ wave in the six groups was: rhEPO hydrogel group, $9.81 \pm 3.85 \mathrm{uV}$; rhEPO group, $3.37 \pm 1.86 u V$; PBS hydrogel group, 3.64 $\pm 2.56 u V ;$ PBS group, $3.08 \pm 1.50 u V ;$ RD group, $1.68 \pm 1.35 \mathrm{uV}$; C57 group, $19.78 \pm 6.77 \mathrm{uV}$. There were no waveforms in the placebo groups and RD group. The value of $b$ wave in rhEPO hydrogel group was higher than that in $\mathrm{RD}$ group $(p=0.025)$. However, there was no significant difference between rhEPO group and RD group.

Figure 5 was the results of $b$ waves of scotopic 3.0 and 10.0 ERG. Figure $5 \mathrm{~A}$ represented the normal waveform in $\mathrm{C} 57$ group. The value of b wave in the six groups was: rhEPO hydrogel group,

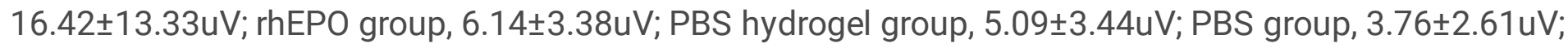
RD group, $4.45 \pm 2.93 \mathrm{uV}$; $C 57$ group, $127.50 \pm 14.73 \mathrm{uV}$. There were no waveforms in the placebo groups and $\mathrm{RD}$ group. The value of $\mathrm{b}$ wave in rhEPO hydrogel group was higher than that in $\mathrm{RD}$ group $(p=0.034)$. However, there was no significant difference between rhEPO group and RD group.

\section{Discussion}

Apoptosis of photoreceptor is the main cause of blindness in the late stage of RP. Moreover, recent studies indicated that in addition to genetic factors, retinal blood supply, oxygen supply, immune factors and optic nerve inflammation and other factors, also have a great impact on retinal degeneration [20-22] And retinal ischemia and hypoxia, oxidative stress is closely related to retinal degeneration. In recent years, EPO has become a safe and effective treatment for retinal disease. Beyond hematopoietic function, its anti-apoptosis and neuroprotective effect has also been proved ${ }^{[11,23]}$.

$\mathrm{Bax}$ and $\mathrm{Bcl}-2$ are a couple members of $\mathrm{Bcl}-2$ family proteins, which are usually used to evaluate apoptosis. The anti-apoptotic effect of EPO in retina is to regulate the expression of $\mathrm{Bax}$ and $\mathrm{Bcl}-2$ through the interaction of EPO/EPOR ${ }^{[24]}$. And the EPOR-mediated extracellular signal-regulated kinase (ERK) is a crucial pathway for anti-apoptosis of EPO ${ }^{[25]}$. Moreover, the PI3K/Akt signal pathway and the 
Erk1/2 signal pathway are essential in neuroprotection. Activating the PI3K/Akt signal pathway and inactivating the Erk1/2 signal pathway to up-regulate the expression of anti-apoptotic factor $\mathrm{Bcl}-2$ and down-regulate the expression of pro-apoptotic factor Bax is the main mechanism of EPO against apoptosis ${ }^{[26]}$. In our present research, mRNA and protein expression of Bax in RD and placebo groups were increased obviously. And mRNA and protein expression of $\mathrm{Bcl}-2$ in these groups were decreased on the contrary. The changes of apoptosis-related factors in RD group indicated the apoptosis of photoreceptor in RD mice. The expression of Bax was down-regulated at two weeks after injection in all rhEPO administrated groups and the expression of $\mathrm{Bcl}-2$ was up-regulated. Our results demonstrated the anti-apoptotic effect of EPO, which were consistent with previous studies ${ }^{[11,27]}$. Furthermore, we made a comparison between rhEPO administration and rhEPO-hydrogel administration, finding that antiapoptotic effect of rhEPO-hydrogel administration group was better than that of the other group. This indicated that the hydrogel we constructed, played a crucial role in lasting administration. However, there was no obvious difference between different routes of administration.

HIF-1a/ iNOS is a crucial signal pathways regulating nerve apoptosis. In vivo, the expression of EPO depends on hypoxia. When the tissue suffered from ischemia-related injury, tissue hypoxia induced hypoxia-inducing factor (HIF-1a) to promote the production of EPO, and had a neuroprotective effect on the injured tissue ${ }^{[28]}$. Previous studies had proved that the expression of HIF-1a had a positive correlation with iNOS ${ }^{[29-30]}$. And EPO could inhibit the expression of HIF-1a and iNOS, when administered in vitro. In our research, we detected the protein and mRNA expression of iNOS, which were decreased in rhEPO administrated groups. Combined with the effect of retinal hypoxia on the progression of RP mentioned above, our results indicated that rhEPO could be used as a neuroprotector in the treatment of rd mice.

Hematopoiesis is the main function of EPO. In Patel's study EPO was related to vascular development, it could promote the development of embryonic retinal vessels, and during the gestation, the mRNA and protein expression of EPO increased gradually ${ }^{[31]}$. Vascular endothelial growth factor (VEGF) was closely associated with EPO in neuroprotection and angiogenesis ${ }^{[32]}$. The expression of EPO was positively correlated with VEGF. VEGFa is one of the founding members of VEGF, which is crucial in blood vessels maintenance ${ }^{[33]}$. Attenuation of blood vessels is one of the typical manifestations of RP fundus, which indicates the poor blood supply of the retina. In our study, the mRNA and protein expression of VEGFa in $\mathrm{RD}$ mice were distinctly decreased, compared with normal ones. After rhEPO administration, the expression levels were up-regulated obviously. This result confirmed the positive correlation between the expression of VEGFa and EPO, illuminated that EPO could protect blood vessels and improve blood supply of retina.

$\mathrm{RP}$ is a chronic lifelong retinal disease, which requires long-term medication to control the progression. Injective drugs like EPO with a half-life period of 6-8 hours, are usually required repeated injection to maintain the effective concentration in the vitreous cavity, which will increase complications of this

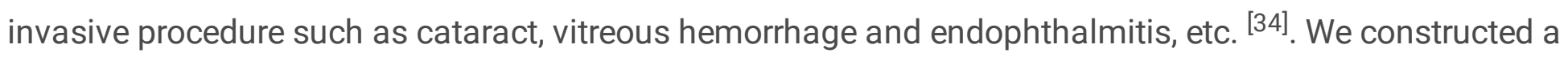


hydrogel drug sustained-release system to alleviate complications caused by repeated injections. From Figure 1, 2 and 3 , the expression of four factors in rhEPO hydrogel group was better than that in rhEPO group, though there was no statistically significant difference between the two groups. Scotopic 0.01 ERG, we recorded, reflects the function of scotopic rod cells. Scotopic 3.0 and 10.0 ERG reflects the function of scotopic cone and rod cells. From Figure 4 and 5 , we can see it intuitively that there were no waveforms in the placebo groups and RD group ERG. Furthermore, there was no significant difference between rhEPO group and RD group in $b$ waves. However, the amplitude of $b$ waves in rhEPO hydrogel group were obviously higher than those in rhEPO group, which indicated that hydrogel loading could more effectively retain the function of photoreceptors, compared with simple drug injection. This evidently proved that CS-PEG hydrogel could maintain the effect of rhEPO for a longer time and sustained-release rhEPO effectively. Previous studies of two kinds of hydrogels, used in the treatment of glaucoma, performed that hydrogels could release drugs stably for a long time ${ }^{[12,15]}$. Our hydrogel was similar to their results in terms of stability. What's more, from the results of ERG, rhEPO groups could give more chance to retain retinal function, which revealed the availability of the hydrogel sustained-release system.

\section{Conclusions}

In conclusion, our present results indicated that administration in the early stage of the disease does delay the progression of RP. EPO was an effective drug in the treatment of RP in terms of anti-apoptosis, neuroprotection and improving blood supply of the retina. The CS-PEG hydrogel sustained release system was stable in storing drugs and effective in sustained releasing drugs. And it might become a potential long-lasting drug delivery system for RP treatment in the futrure.

\section{Declarations}

\section{Abbreviations}

RP: Retinitis pigmentosa; rhEPO: recombinant human erythropoietin; rd: retinal degenerative; ERG: Electroretinogram; RPE: retinal pigment epithelium; EPO: erythropoietin; EPOR: EPO receptors; IPL: inner plexiform layer; OPL: outer plexiform layer; GCL: ganglion cells layer; ILM: inner limiting membrane; DR: diabetic retinopathy; AMD: age-related macular degeneration; MNU: N-methyl-N-nitrosourea; IOP: intraocular pressure; CS: chitosan; PEG: polyethylene glycol; PVDF: polyvinylidene difluoride; TBST: TrisBuffered Saline and Tween 20; qRT-PCR: quantitative reverse transcription-polymerase chain reaction; LED: light emitting diode; SD: standard deviation; ERK: extracellular signal-regulated kinase; HIF-1a: hypoxia-inducing factor; VEGF: Vascular endothelial growth factor.

\section{Ethics approval and consent to participate}

The C57BL/6 mice were purchased from HFK Bioscience Co. Ltd (Beijing, China). The rd (Pdeb ${ }^{\text {rd1/ }}$ $\mathrm{Pdeb}^{\mathrm{rd} 1}$ ) mice were purchased from The Jackson Laboratory (USA) and housed in the experimental 
facility (room temperature at about $25^{\circ} \mathrm{C}$, on a 12-hour light/dark cycle) at the department of laboratory animal, Capital Medical University. The breeding grade was SPF (Specific Pathogen Animal). The care and maintenance of the mice were referred to the ARVO guidelines. The experiment was approved by the Animal Experiment Ethics Committee of Capital Medical University (NO. AEEI-2019-033). The mice were euthanized by an overdose of anesthesia $(0.3 \%$ sodium pentobarbital, $0.15 \mathrm{ml} / 10 \mathrm{~g}$, intraperitoneal injection).

\section{Consent for publication}

Not applicable.

\section{Availability of data and material}

The datasets generated and analysed during the current study are available from the corresponding author on reasonable request.

\section{Competing interests}

All authors declare that they have no conflicts of interest regarding this submission.

\section{Funding}

This study was financed by a grant from the National Natural Science Foundation of China (No. 81271046) in mice breeding, financial support for the experiment and data analysis.

\section{Authors' contributions}

Conceived and designed the experiments: GL LY. Performed the experiments: ZZ HD RL YL. Analyzed the data: ZZ HD. Contributed reagents/materials/analysis tools: ZZ. Wrote the paper: ZZ. All authors have read and approved the final manuscript.

\section{Acknowledgments}

Not applicable

\section{References}


[1] You Q S, Xu L, Wang Y X, et al. Prevalence of retinitis pigmentosa in North China: the Beijing Eye Public Health Care Project. Acta Ophthalmologica. 2013; 91(6): e499-e500.

[2] Russell S, Bennett J, Wellman JA, et al. Efficacy and safety of voretigene neparvovec (AAV2hRPE65v2) in patients with RPE65-mediated inherited retinal dystrophy: a randomised, controlled, openlabel, phase 3 trial. Lancet. 2017; 390(10097): 849-860.

[3] Daiger SP, Sullivan LS, Bowne SJ. Genes and mutations causing retinitis pigmentosa. Clin Genet. 2013; 84(2): 132-141.

[4] Fisher JW. Erythropoietin: physiology and pharmacology update. Exp Biol Med (Maywood). 2003; 228(1): 1-14.

[5] Hardee ME, Arcasoy MO, Blackwell KL, et al. Erythropoietin biology in cancer. Clin Cancer Res. 2006; 12(2): 332-9.

[6] S B, M M, Corsini E GC, B V. Erythropoietin: a novel neuroprotective cytokine. Neurotoxicology. 2005; 26(5): 923-8.

[7] Gawad AE, Schlichting L, Strauss O, et al. Antiapoptotic properties of erythropoietin: novel strategies for protection of retinal pigment epithelial cells. Eye (Lond). 2009; 23(12): 2245-50..

[8] Feng Q. Beyond erythropoiesis: the anti-inflammatory effects of erythropoietin. Cardiovasc Res. 2006; 71(4): 615-7.

[9] Munro K, Rees S, O'Dowd R, et al. Developmental profile of erythropoietin and its receptor in guinea-pig retina. Cell Tissue Res. 2009; 336(1): 21-9.

[10] Abri Aghdam K, Soltan Sanjari M, Ghasemi Falavarjani K. Erythropoietin in ophthalmology: A literature review. Journal of Current Ophthalmology. 2016; 28(1): 5-11.

[11] Tao Y, Wang Y, Ma Z, et al. Subretinal delivery of erythropoietin alleviates the N-methyl-Nnitrosourea-induced photoreceptor degeneration and visual functional impairments: an in vivo and ex vivo study. Drug Delivery. 2017; 24(1): 1273-1283.

[12] Cheng $\mathrm{YH}$, Hung $\mathrm{KH}$, Tsai TH, et al. Sustained delivery of latanoprost by thermosensitive chitosangelatin-based hydrogel for controlling ocular hypertension. Acta Biomater. 2014; 10(10): 4360-4366.

[13] Song Y, Nagai N, Saijo S, et al. In situ formation of injectable chitosan-gelatin hydrogels through double crosslinking for sustained intraocular drug delivery. Materials Science \& Engineering C Materials for Biological Applications. 2018; 88:1.

[14] Gao Y, Sun Y, Ren F, et al. PLGA-PEG-PLGA hydrogel for ocular drug delivery of dexamethasone acetate. Drug Development \& Industrial Pharmacy. 2010; 36(10): 1131-1138. 
[15] Natesan S, Pandian S K, Ponnusamy C, et al. Co-encapsulated resveratrol and quercetin in chitosan and peg modified chitosan nanoparticles: for efficient intra ocular pressure reduction. International Journal of Biological Macromolecules. 2017; 104: 1837-1845.

[16] Petersen-Jones S M. Animal models of human retinal dystrophies. Eye. 1998; 12 (Pt 3b)(3b): 566.

[17] Strettoi E, Pignatelli V. Modifications of retinal neurons in a mouse model of retinitis pigmentosa. Proceedings of the National Academy of Sciences. 2000; 97(20): 11020-11025.

[18] Mclaughlin M E, Sandberg M A, Berson E L, et al. Recessive mutations in the gene encoding the $\beta$-subunit of rod phosphodiesterase in patients with retinitis pigmentosa. Nature Genetics. 1993; 4(2): 130-134.

[19] Duan X, Li G, Wang J. Investigation of the early morphological changes of photoreceptor segment in animal model of hereditary retinal degeneration. Ophthalmol CHN. 2003; 12(4): 237-240.

[20] Nussenblatt RB, Liu B, Wei L, Sen HN. Int Rev Immunol. The Immunological Basis of Degenerative Diseases of the Eye. 2013; 32(1): 97-112.

[21] Madeira MH, Boia R, Santos PF, et al. Contribution of Microglia-Mediated Neuroinflammation to Retinal Degenerative Diseases. Mediators Inflamm. 2015; 2015: 673090.

[22] Battu R, Mohan A, Khanna A, et al. Retinal oxygen saturation in retinitis pigmentosa and macular dystrophies in asian-Indian eyes. Invest Ophthalmol Vis Sci. 2015; 56(5): 2798-2802.

[23] Luo W, Hu L, Wang F. The Protective Effect of Erythropoietin on the Retina. Ophthalmic Research. 2015; 53(2): 74-81.

[24] Nekoui A, Blaise G. Erythropoietin and Nonhematopoietic Effects[J]. American Journal of the Medical Sciences. 2017; 353(1): 76-81.

[25] Hernández C, Simó R. Erythropoietin produced by the retina: its role in physiology and diabetic retinopathy. Endocrine. 2012; 41(2): 220-226.

[26] Si W, Wang J, Li M, et al. Erythropoietin protects neurons from apoptosis via activating PI3K/AKT and inhibiting Erk1/2 signaling pathway. 3 Biotech. 2019; 9(4): 131.

[27] Zou YR, Zhang J, Wang J, et al. Erythropoietin receptor activation protects the kidney from ischemia/reperfusion-induced apoptosis by activating ERK/p53 signal pathway. Transplant Proc. 2016; 48(1): 217-21. $\otimes$

[28] Grimm C, Wenzel A, Acar N, et al. Hypoxic preconditioning and erythropoietin protect retinal neurons from degeneration. Adv Exp Med Biol. 2006; 588: 119-31. 
[29] Gui D, Li Y, Chen X, et al. HIF 1 signaling pathway involving iNOS, COX 2 and caspase 9 mediates the neuroprotection provided by erythropoietin in the retina of chronic ocular hypertension rats. Molecular Medicine Reports. 2015; 11(2): 1490.

[30] Lee M, Wang C, Jin S W, et al. Expression of human inducible nitric oxide synthase in response to cytokines is regulated by hypoxia-inducible factor-1. Free Radic. Biol. Med. 2019; 130: 278-287.

[31] Patel S, Rowe MJ, Winters SA, et al. Elevated Erythropoietin mRNA and Protein Concentrations in the Developing Human Eye. Pediatr Res. 2008; 63(4): 394-7.

[32] Stahl A, Buchwald A, Martin G, et al. Vitreal levels of erythropoietin are increased in patients with retinal vein occlusion and correlate with vitreal VEGF and the extent of macular edema.Retina (Philadelphia, Pa.). 2010; 30: 1524-9.

[33] Staels W, Heremans $\mathrm{Y}$, Heimberg $\mathrm{H}$, et al. VEGF-A and blood vessels: a beta cell perspective. Diabetologia. 2019.

[34] Rong X, Mo X, Ren T, et al. Neuroprotective effect of erythropoietin-loaded composite microspheres on retinal ganglion cells in rats. Eur J Pharm Sci. 2011; 43(4): 334-42.

\section{Figures}




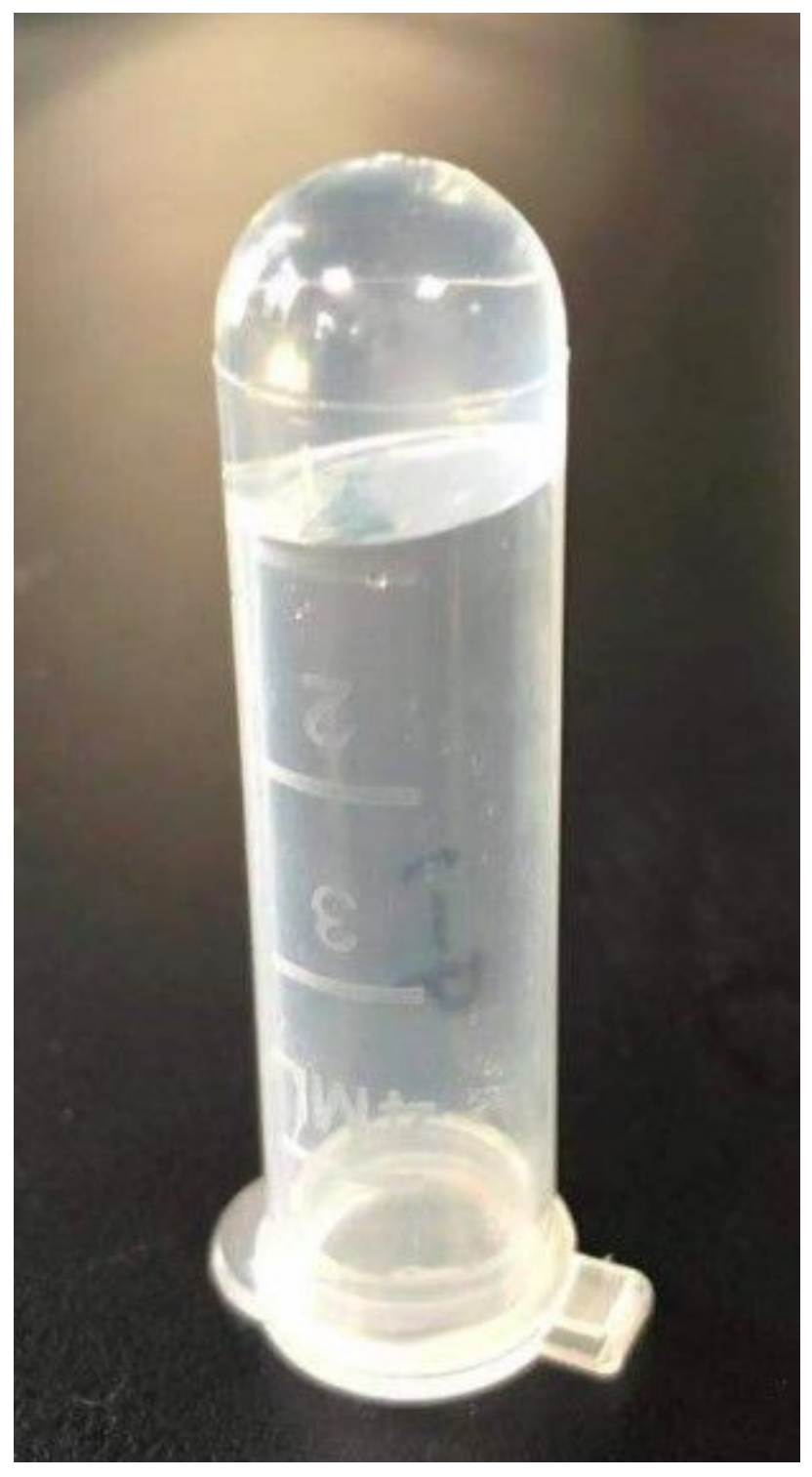

Figure 1

CS-PEG hydrogel of rhEPO.

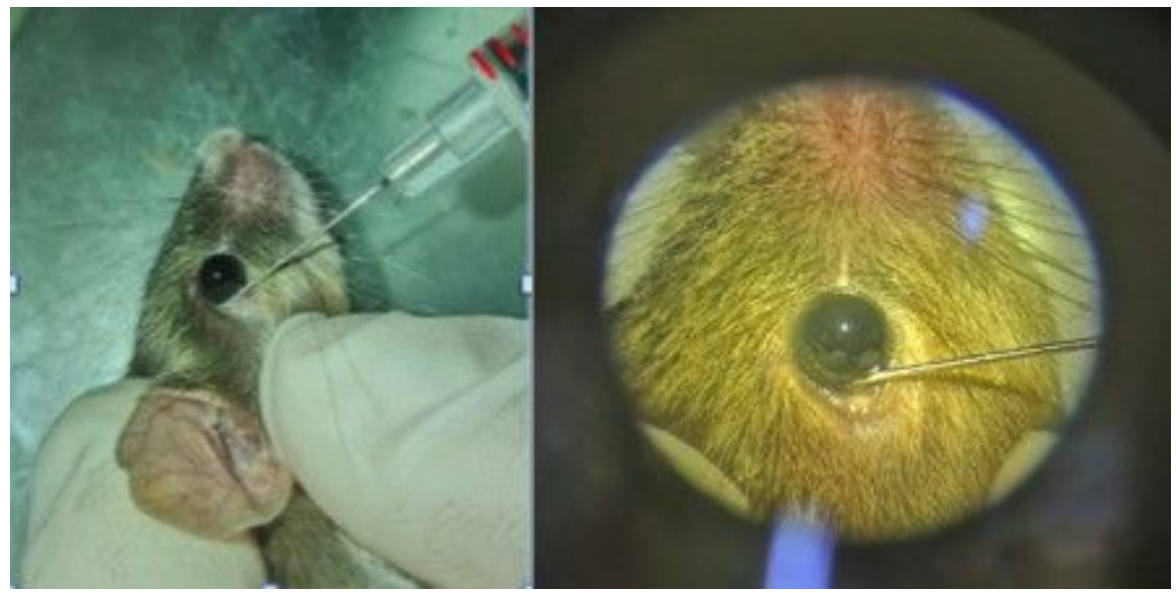

Figure 2 
Subconjunctival injection of rhEPO/rhEPO hydrogel in mice.
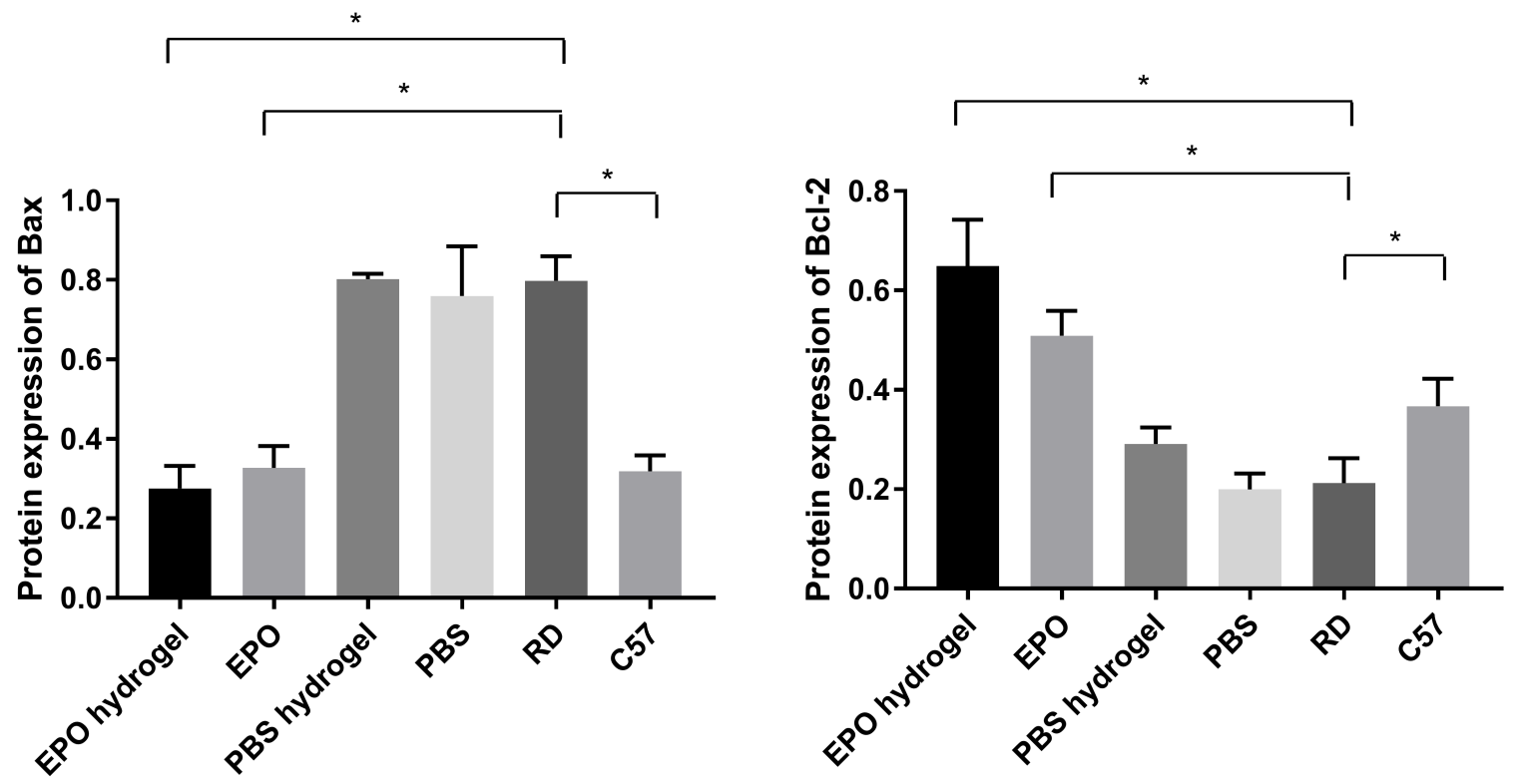

(A)

(B)

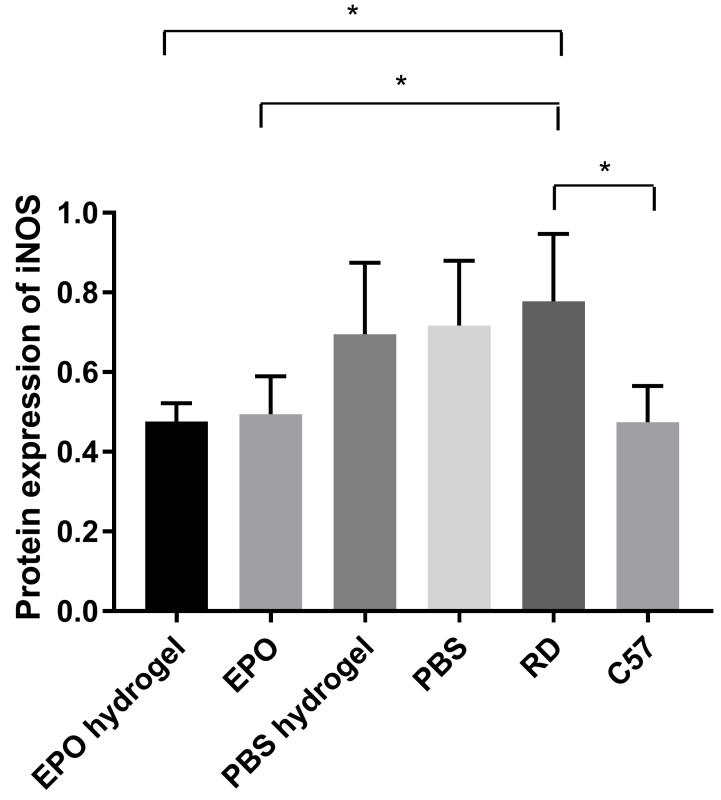

(C)

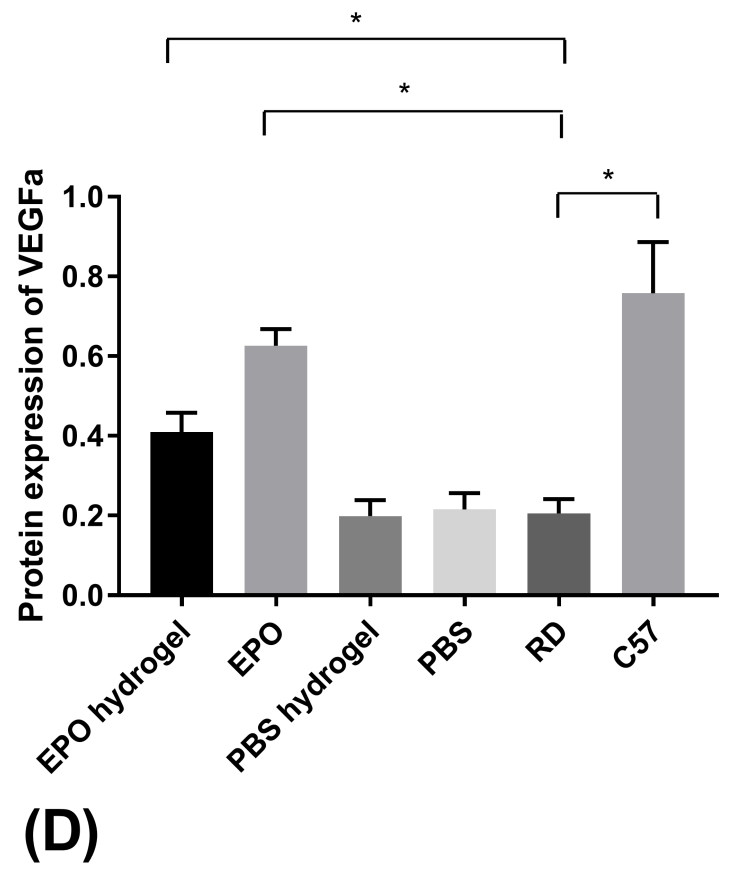

Figure 3

The protein expression of Bax, Bcl-2, iNOS and VEGFa of each group at PN-4w. (mean $\pm S D$ ). (A) The mRNA expression of Bax in RD group was obviously higher than that in $\mathrm{C} 57$ group. There was no significant difference between placebo groups and RD group. The expression in rhEPO administrated groups were decreased, compared with RD group. (B) The mRNA expression of Bcl-2 in RD group was 
obviously lower than that in $\mathrm{C} 57$ group. There was no significant difference between placebo groups and $\mathrm{RD}$ group. The expression in rhEPO administrated groups were increased, compared with RD group. (C) The mRNA expression of iNOS in RD group was higher than that in C57 group. There was no significant difference between placebo groups and RD group. The expression in rhEPO administrated groups were decreased, compared with RD group. (D) The mRNA expression of VEGFa in RD group was obviously lower than that in $\mathrm{C} 57$ group. There was no significant difference between placebo groups and RD group. The expression in rhEPO administrated groups were increased, compared with RD group. * represents significant difference between the two groups. $(p<0.05)$.

Bax
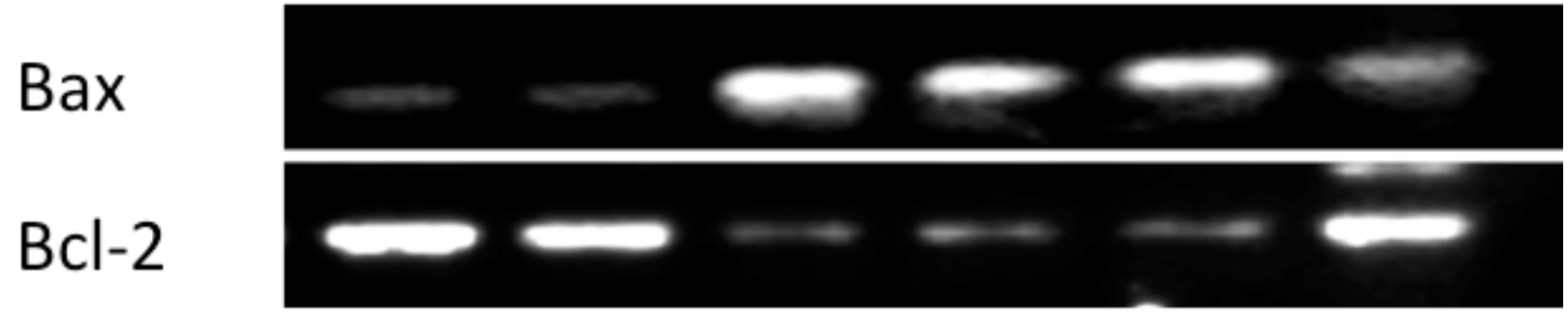

\section{iNOS}

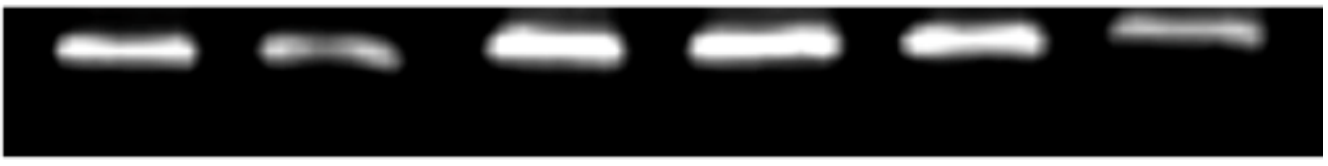

\section{VEGFa}

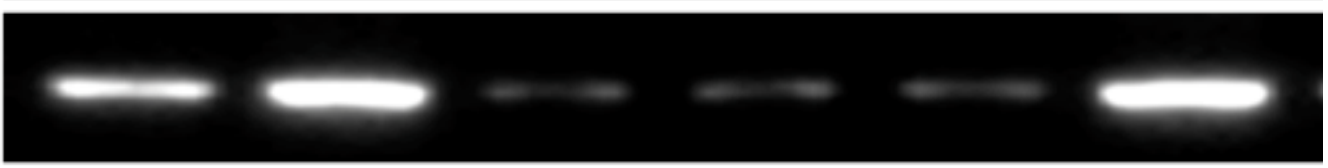

\section{GAPDH}

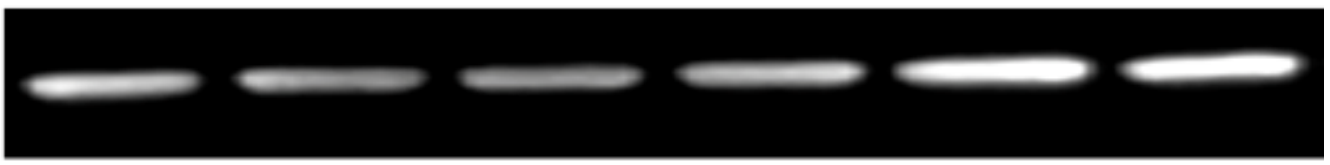

\section{Figure 4}

The protein expression of factors in each group at PN-4w. The six groups from left to right are: rhEPO hydrogel group, rhEPO group, PBS hydrogel group, PBS group, RD group and C57 group. 

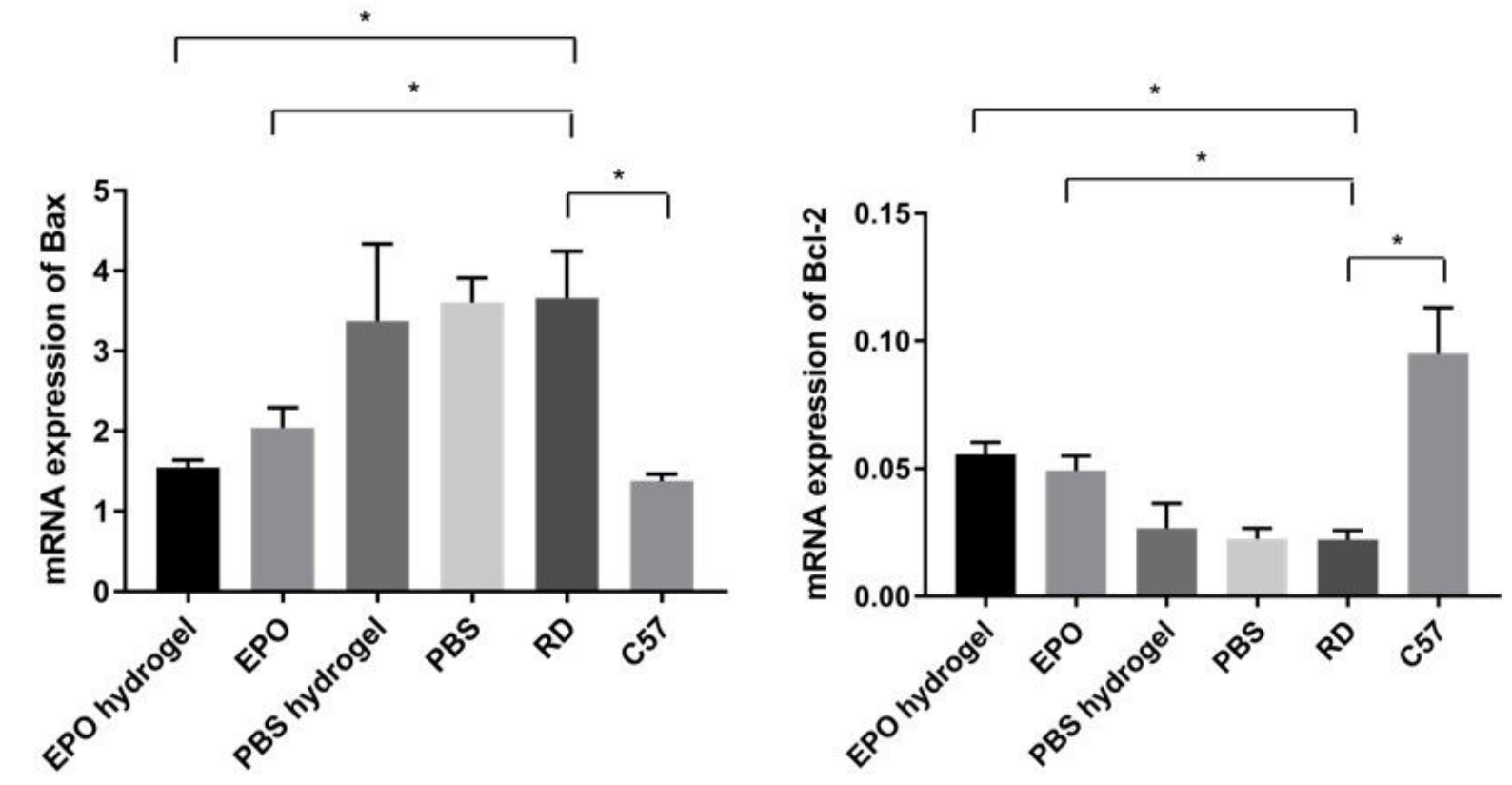

(A)

(B)
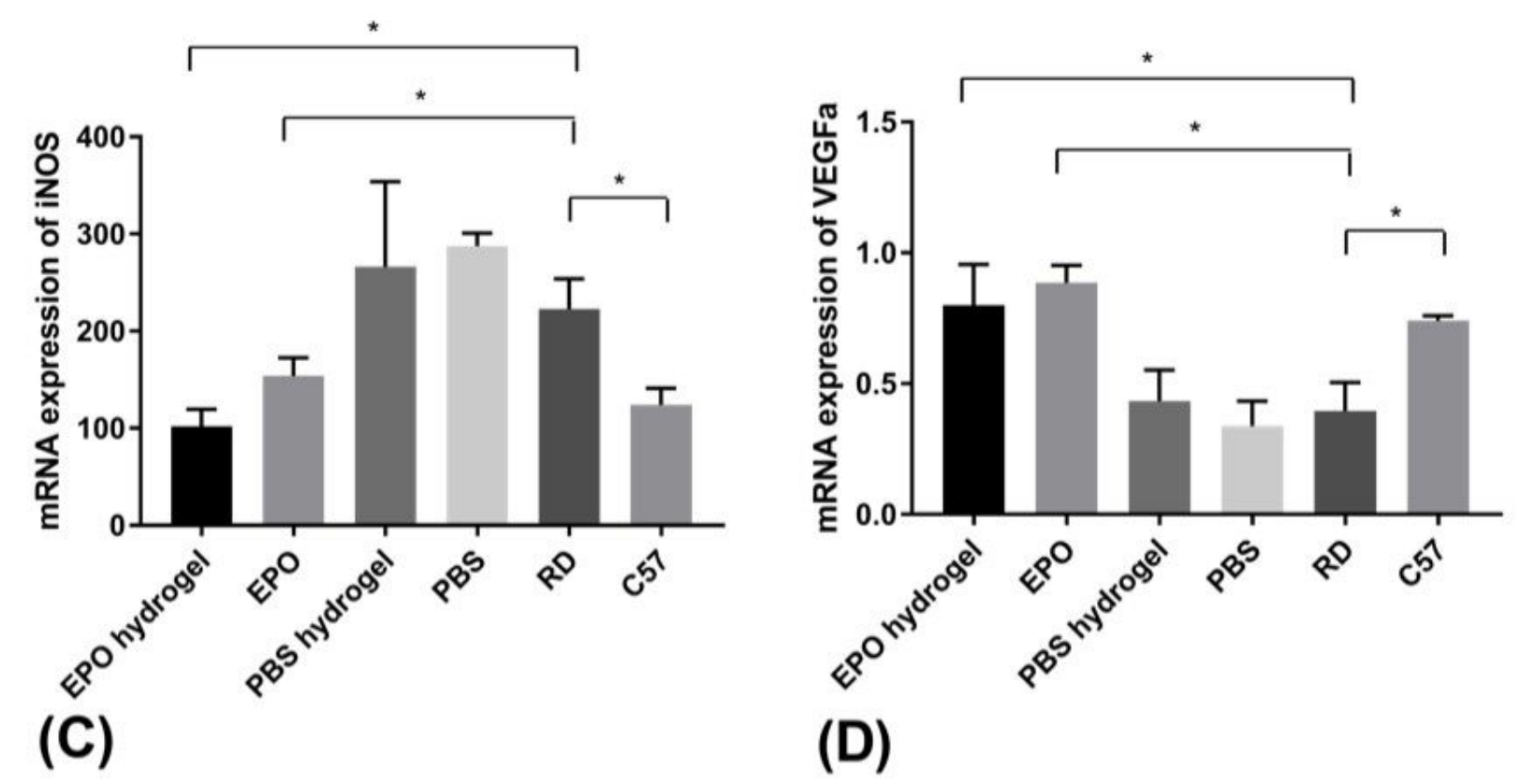

Figure 5

The mRNA expression of Bax, Bcl-2, iNOS and VEGFa of each group at PN-4w. (mean $\pm S D$ ). (A) The mRNA expression of Bax in RD group was obviously higher than that in $\mathrm{C} 57$ group. There was no significant difference between placebo groups and RD group. The expression in rhEPO administrated groups were decreased, compared with RD group. (B) The mRNA expression of Bcl-2 in RD group was obviously lower than that in $\mathrm{C} 57$ group. There was no significant difference between placebo groups and RD group. The 
expression in rhEPO administrated groups were increased, compared with RD group. (C) The mRNA expression of iNOS in RD group was higher than that in C57 group. There was no significant difference between placebo groups and RD group. The expression in rhEPO administrated groups were decreased, compared with RD group. (D) The mRNA expression of VEGFa in RD group was obviously lower than that in $\mathrm{C} 57$ group. There was no significant difference between placebo groups and RD group. The expression in rhEPO administrated groups were increased, compared with RD group. * represents significant difference between the two groups. $(p<0.05)$.
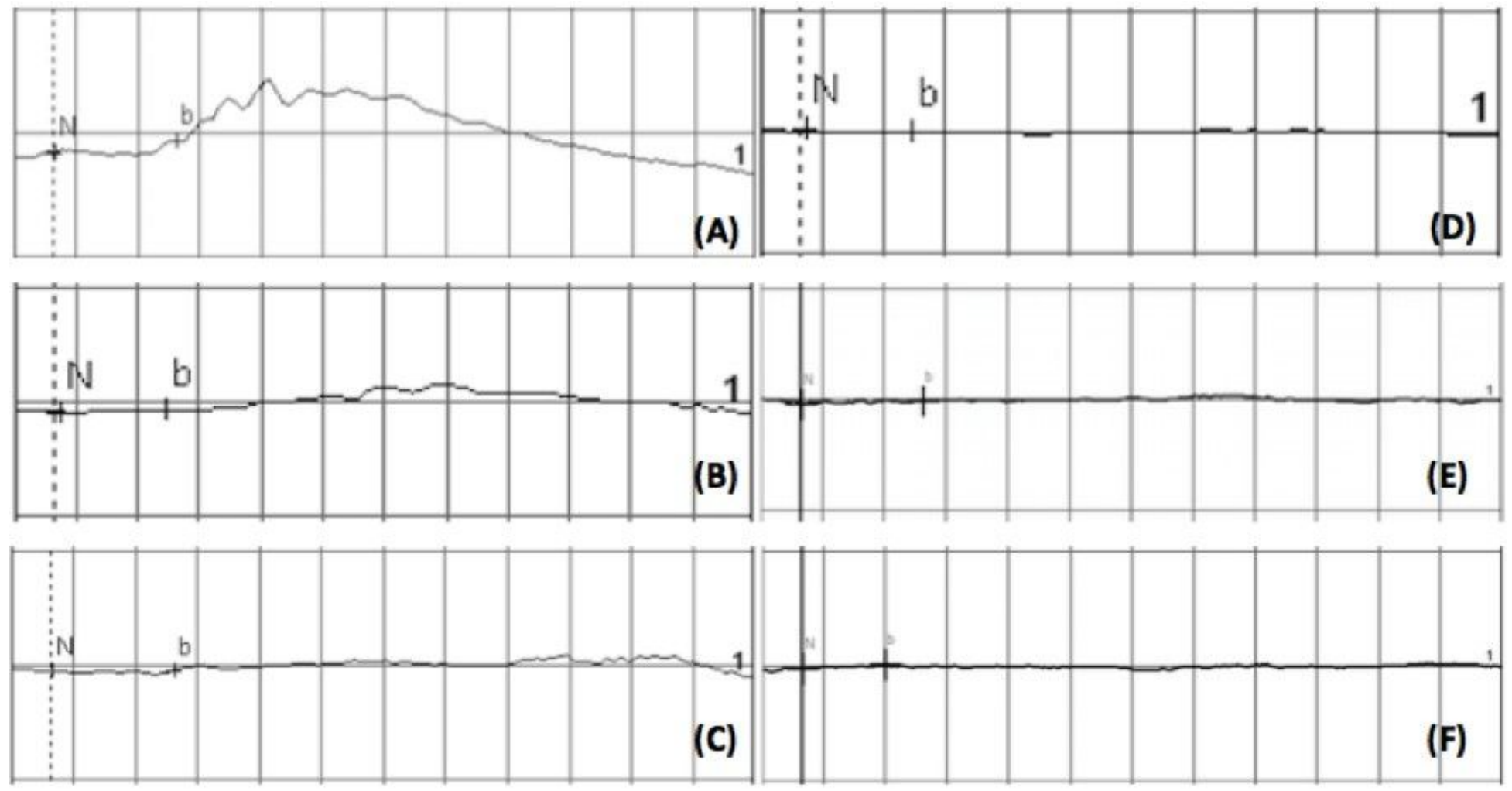

\section{Figure 6}

The Scotopic 0.01 ERG wave forms of each group at PN-4w. (A) C57 (normal) group. (B) rhEPO hydrogel group. (C) rhEPO group. (D) PBS hydrogel group. (E) PBS group. (F) RD (control) group. 

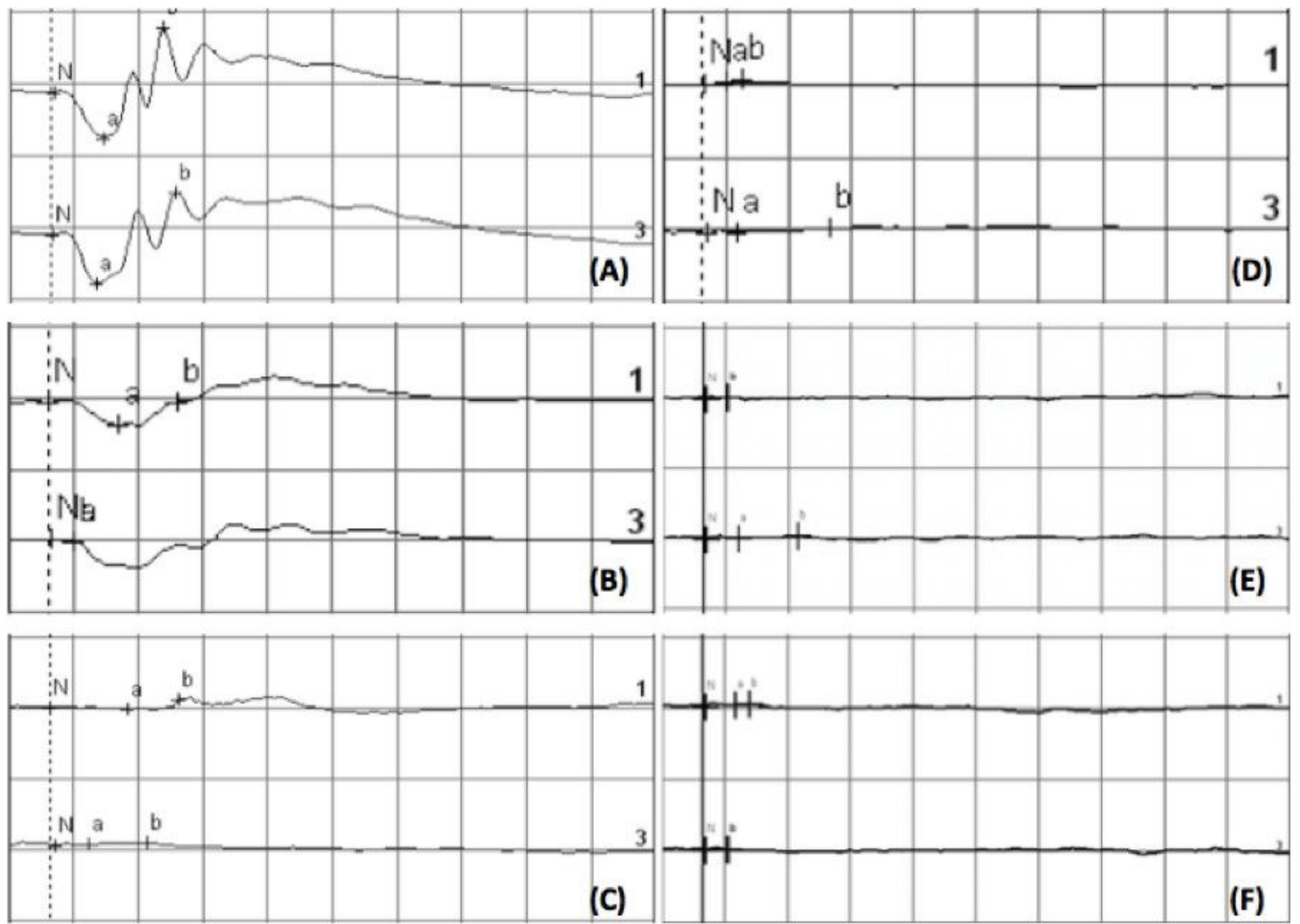

Figure 7

The Scotopic 3.0 and 10.0 ERG wave forms of each group at PN-4w. (A) C57 (normal) group. (B) rhEPO hydrogel group. (C) rhEPO group. (D) PBS hydrogel group. (E) PBS group. (F) RD (control) group.

\section{Supplementary Files}

This is a list of supplementary files associated with this preprint. Click to download.

- NC3RsARRIVEGuidelinesChecklistfillable.pdf 